\title{
Hydrogen Permeation Behavior of Stainless Steels Treated with Nitrogen-Plasma
}

\author{
Motonori Tamura \\ Center for Industrial and Governmental Relations, The University of Electro-Communications, Tokyo 182-8585, Japan
}

\begin{abstract}
The surface of austenitic stainless steel SUS316L was treated with nitrogen-plasma and its hydrogen permeability function was investigated. On the stainless steel surface, a nitride phase and a nitrogen-dispersed phase were found $5 \mu \mathrm{m}$ from the surface. The nitride phase was mainly consisting of $\varepsilon-\mathrm{Fe}_{2-3} \mathrm{~N}$ particles $0.1-0.4 \mu \mathrm{m}$ in size. This nitride phase had hydrogen permeability an order of magnitude less than that for the untreated sample. The plasma nitride sample was confirmed to have a hydrogen permeation prevention effect.
\end{abstract}

Key words: Hydrogen permeation, stainless steel, nitride phase, nitrogen-plasma.

\section{Introduction}

High-strength steel and various structural metals such as $\mathrm{Al}, \mathrm{Ti}$ and $\mathrm{Zr}$ are likely to undergo hydrogen embrittlement due to penetration by hydrogen in the environment in which it is used, such as a corrosive atmosphere, high-temperature water, sour gas, and high-pressure hydrogen gas. Because of recent trends in terms of further reinforcement of high-strength steel or the demand to ensure the safety of container materials for high-pressure hydrogen gas following the promotion of hydrogen energy, the topic of hydrogen embrittlement is again becoming an issue of concern.

One of the ways to prevent hydrogen embrittlement that is being researched now is the formation of a hydrogen barrier, which is obtained by coating the metal surface with a ceramic film, thus delaying penetration by hydrogen. For example, films such as $\mathrm{Al}_{2} \mathrm{O}_{3}$, TiN, TiC, and $\mathrm{BN}$ are efficient hydrogen barriers [1-3]. Films created using methods such as PVD show better adhesion with the metal surface, have fewer inner vacancies or particles, and are known to be suitable for fine and smooth surfaces.

Corresponding author: Motonori Tamura, Ph.D., professor, research fields: metallurgical coatings.
The development of fuel cells involving hydrogen and the use of hydrogen energy will continue progress, and various materials are expected to come into contact with hydrogen in various ways, such as during the production of hydrogen and in its storage, transport, and use. Thus, techniques for making the surfaces of various types of substrates function as hydrogen barriers are desired. The PVD process results in the formation of films with strong directionality, but it has limited applications in the case of complex shapes, the inner surfaces of pipes, or medium/large-sized components.

In this research, surface-treatment processes using various gases that can impart hydrogen barrier functionality to the surfaces of substrates of various shapes were studied. The focus of the study was particularly on plasma-nitride treatment. Austenite-based stainless steel SUS316L is a material recommended for use with hydrogen [4], but a SUS316L stainless steel substrate forms a passive film on the surface. The nitride treatment has to be carried out by allowing nitrogen to impregnate the steel after removal of the passive film. Conventional nitride treatment consists of removing the passive layer by pre-treatment at high temperature or salt-bath nitride. When austenite-based stainless steel is held at a 
temperature exceeding $873 \mathrm{~K}$, there is a possibility of deformation of the substrate or loss of corrosion resistance of the stainless steel owing to the formation of Cr-based nitride. Furthermore, the salt-bath method has the problem of emission of toxic gases. In contrast, the plasma-nitride process allows for the removal of the passive film at a temperature up to 873 $\mathrm{K}$ and without the use of any toxic gas [5]. The relationship between plasma nitride and hydrogen permeation has not been explored sufficiently.

This research explores hydrogen permeation behavior of austenitic stainless steels treated by nitrogen plasma.

\section{Experimental Setup}

\subsection{Surface-Nitride Process}

Plasma nitride was carried out using glow discharge in a vacuum chamber by connecting the stainless steel substrate to a biased power source. The conditions for the treatment are depicted in Table 1. Nitrogen and hydrogen were used as the gases and the ratio of flow rates of nitrogen and hydrogen was 7:3. The substrate was austenite-based SUS316L stainless steel with an outer diameter of $35 \mathrm{~mm}$ and a thickness of $0.1 \mathrm{~mm}$. Glow discharge enhances the removal of the oxide film on the base material, heating, the dispersion of ions or atoms, and the formation of nitride. The surface of the sample was inspected with an infrared temperature sensor and care was taken that the surface temperature of the sample did not increase over 773 $\mathrm{K}$. The bias voltage was $400-600 \mathrm{~V}$.

\subsection{Characterization of the Surface-Nitride Stainless} Steels

The methods used for the characterization of surface-nitride stainless steels were as follows.

Table 1 Surface-nitride conditions.

\begin{tabular}{lllll}
\hline \multirow{2}{*}{ No. } & $P$ & Gas & $T_{\text {sub }}$ & Time \\
\cline { 2 - 5 } & $(\mathrm{Pa})$ & $\left(\mathrm{N}_{2}: \mathrm{H}_{2}\right)$ & $(\mathrm{K})$ & $(\mathrm{h})$ \\
\hline 1 & 180 & $7: 3$ & 673 & 4 \\
2 & 260 & $7: 3$ & 723 & 7 \\
\hline
\end{tabular}

The analysis of the crystalline phase was carried out by the X-ray diffraction method (XRD). The angle of incidence was $1^{\circ}$. Observation of structure of the surface and cross section of the samples was performed using an optical microscope (OM) and a scanning electron microscope (SEM). In the observation of structure of the cross section by using an optical microscope, the sample was embedded in resin, polished, and observed after oxalic acid etching. For compositional analysis of the cross section of the film, an electron probe micro-analyzer (EPMA) was used.

\subsection{Hydrogen-Permeation Tests}

Fig. 1 shows a schematic illustration of the apparatus. Hydrogen-permeation tests were performed on the coated stainless steel samples and surface-nitride stainless steel samples. These tests were based on the differential-pressure methods described in ISO15105-1: 2007, the International Standard for Determination of Gas Transmission Rates [6-8]. Fig. 1 shows a schematic of the apparatus. The coated samples were set on a susceptor made of silicon fiberglass, such that they could be held in place without bending at temperatures up to $773 \mathrm{~K}$. The apparatus was evacuated to $\sim 10^{-6} \mathrm{~Pa}$. After the test temperature had become stable, hydrogen (purity of 99.995\%) was introduced into one side (susceptor side) and subjected to increasing filling pressures (400 $\mathrm{Pa}$. The other side of the apparatus (stainless-steel-sample side) was continuously evacuated. The samples were affixed with a metallic gold seal and heated by an electric furnace to $773 \mathrm{~K}$ without oxidation. The permeation area was $6.6 \mathrm{~cm}^{2}$.

The permeability, $\phi$ is generally defined by the expression $[6,9]$

$$
\phi=(J d) /\left(A \Delta p^{n}\right)
$$

where, $J$ is the permeation flux of hydrogen through a sample of area $A$ and thickness $d$ under a partial pressure gradient, $\Delta p$ across the sample, called the driving pressure. The exponent $n$ represents different 


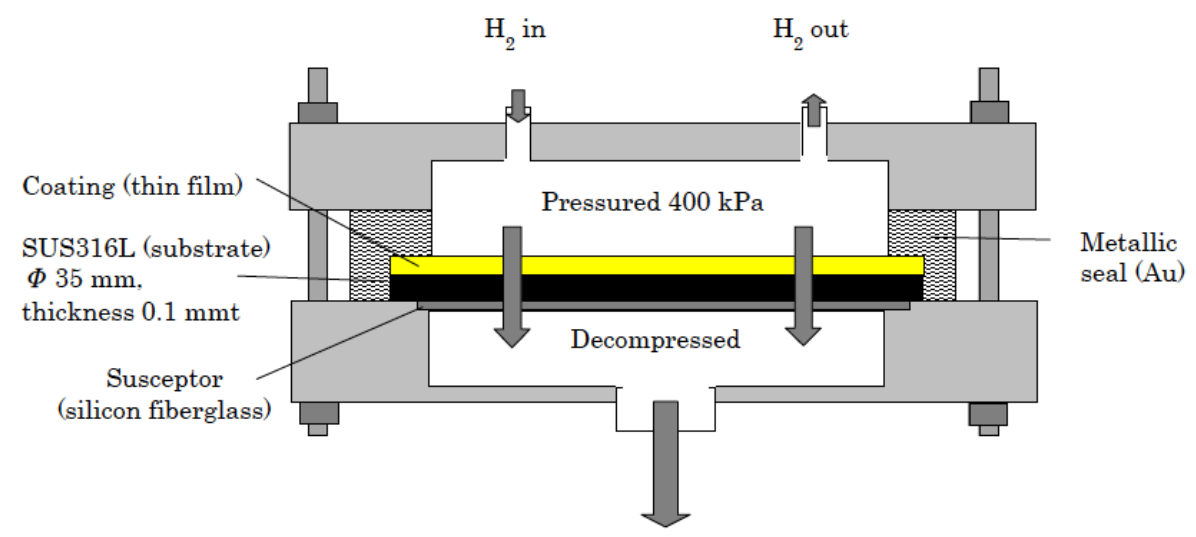

Measured by gas chromatography

Fig. 1 Apparatus for hydrogen-permeation tests.

permeation regimes: diffusion-limited and surface-limited when $n=0.5$ and 1 , respectively. It is known that hydrogen permeation through thin-film-coated steel samples is diffusion-limited when the driving pressure is between $10^{4}$ and $10^{5} \mathrm{~Pa}$ $[3,9]$.

The permeation flux was continuously measured at a test temperature. After $30 \mathrm{~min}$, the standard deviation decreased to less than $10 \%$ among the data for the permeation flux through the non-coated stainless steel substrate at $773 \mathrm{~K}$ under a hydrogen pressure of $400 \mathrm{KPa}$.

\section{Results and Discussion}

\subsection{Microstructure of the Surface-Nitride Stainless} Steels

Fig. 2 shows the results of the XRD analysis of the surface-nitride stainless steel surface. In surface nitride 1 as shown in Fig. 2b, the formation of $\varepsilon-\mathrm{Fe}_{2-3} \mathrm{~N}$ and $\gamma-\mathrm{Fe}_{4} \mathrm{~N}$ was observed. The formation of $\varepsilon-\mathrm{Fe}_{2-3} \mathrm{~N}$ and $\gamma-\mathrm{Fe}_{4} \mathrm{~N}$ was also observed in surface nitride 2 as shown in Fig. 2c; however, each of the peaks was more distinct, indicating that the formation and crystallization of nitride was in progress.

Fig. 3 shows optical microscope images of the cross sections of the samples. In surface nitride 1 as shown in Fig. 3b, the formation of a nitride phase $5 \mu \mathrm{m}$ thick on the surface and back face of the stainless steel was confirmed. In surface nitride 2 as shown in Fig. 3c, this nitride phase was found to be thicker $(8 \mu \mathrm{m})$ and its surface was found to be more uneven than in case of surface nitride 1 .

Fig. 4 shows SEM images of the surfaces of the sample. In surface nitride 1 as shown in Fig. 4b, the

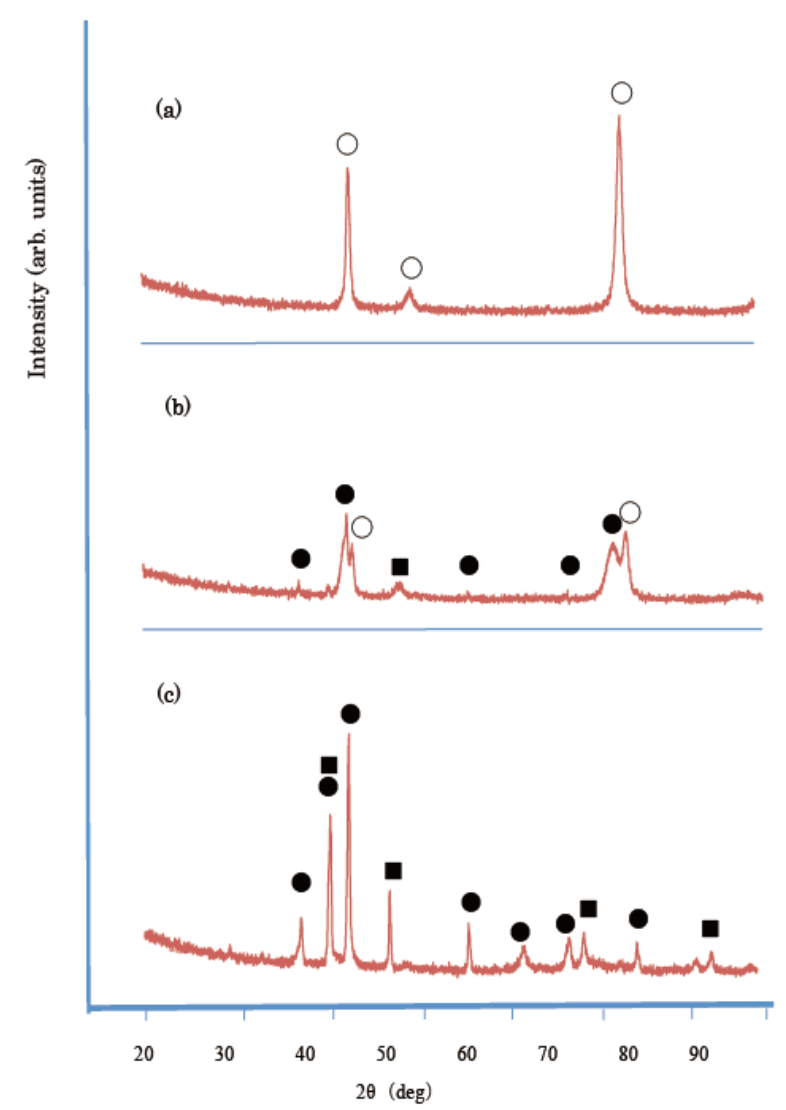

Fig. 2 XRD patterns of (a) SUS316L, (b) surface-nitride 1, and (c) surface-nitride $2 . \bigcirc \alpha-\mathrm{Fe} \bullet \varepsilon-\mathrm{Fe}_{2-3} \mathrm{~N}=\gamma-\mathrm{Fe}_{4} \mathrm{~N}$. 


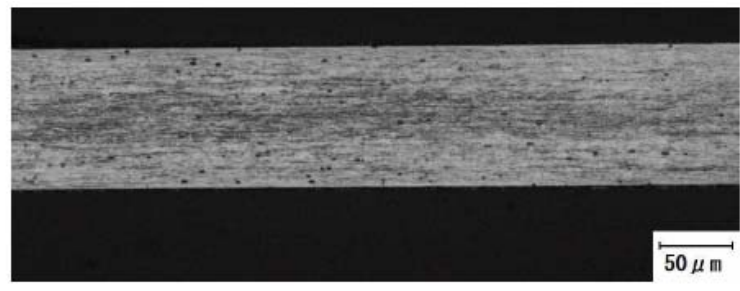

(a)

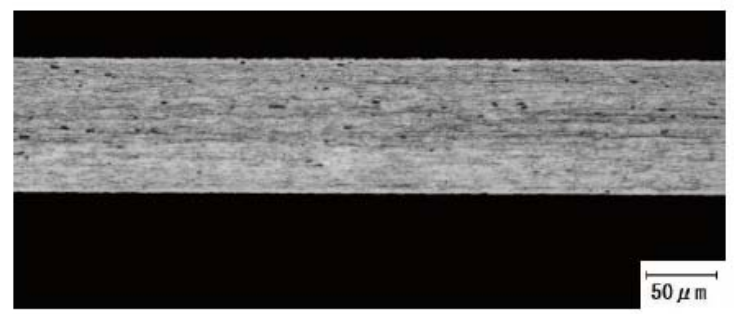

(b)

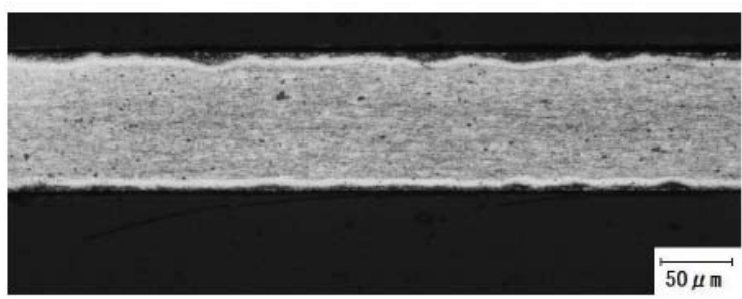

(c)

Fig. 3 Optical microscope images showing cross section of (a) SUS316L, (b) surface-nitride 1, and (c) surface-nitride 2.

size of the nitride grains increased to 0.1-0.4 $\mu \mathrm{m}$. In surface nitride 2 as shown in Fig. 4c, the size of nitride grains increased to $0.3-1.1 \mu \mathrm{m}$.

From these results and observations and EPMA analysis of the fractured surfaces, it was found that in the case of surface nitride 1, iron nitride had formed 1 $\mu \mathrm{m}$ below the surface layer and that approximately 4 $\mu \mathrm{m}$ of a $20-40 \%$ nitrogen-abundant $\mathrm{Fe}-\mathrm{N}$ solid solution (nitrogen-dispersed phase) had formed below that. In surface nitride 2, the iron nitride compound had formed $1.8 \mu \mathrm{m}$ below the surface layer, and below that, a $20-40 \%$ nitrogen-abundant Fe-N solid solution approximately $6 \mu \mathrm{m}$ thick had formed, similar to that in the case of surface nitride 1 . This nitrogen-abundant Fe-N solid solution is a nitrogen-dispersed phase and nitrogen supersaturated solid solution, a nitrogen-S phase, which is known to improve the abrasion resistance of stainless steel without losing corrosion resistance [10-12].

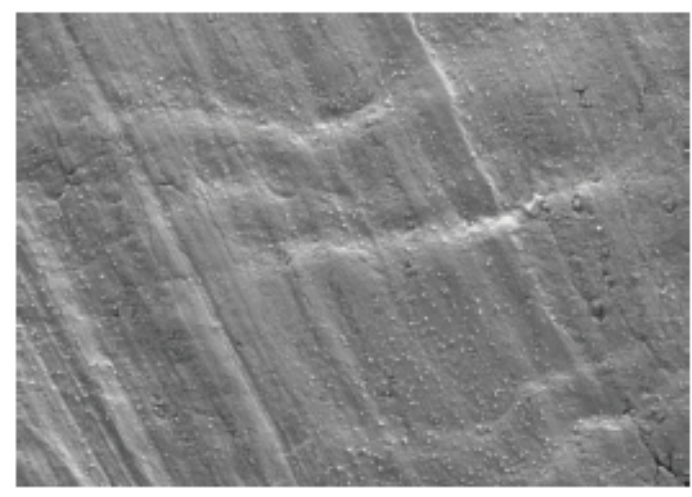

(a)

$1 \mu \mathbf{m}$

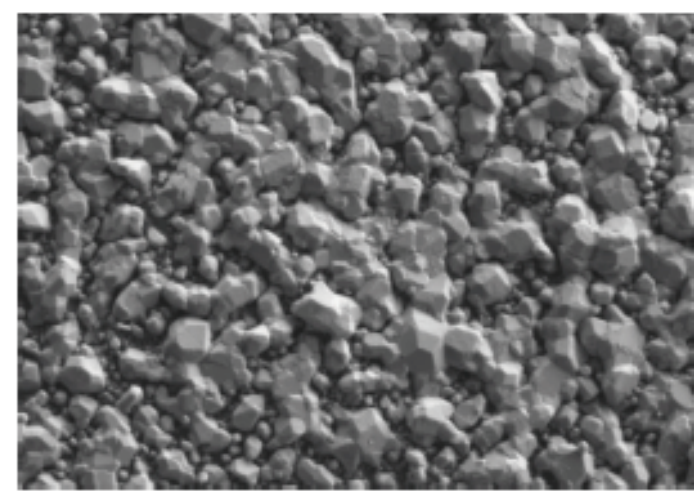

(b)

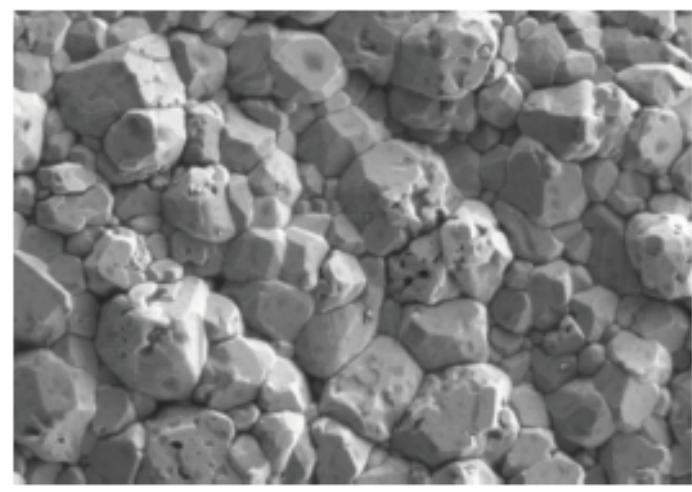

(c)

Fig. 4 SEM images showing surface morphologies of (a) SUS316L, (b) surface-nitride 1, and (c) surface-nitride 2.

Carburizing and nitride treatment have been known to improve the quality of the surface of stainless steel, including iron and steel materials, for a long time. These are methods in which only the surface is hardened, without the loss of toughness of the iron or the steel material itself, which improves the abrasion resistance and fatigue characteristics. As compared to 
nitride methods such as salt-bath nitride or gas-nitride methods, plasma nitride is non-polluting method, and it does not involve the discharge of polluted water. Because the treatment is performed in an activated nitrogen atmosphere, the nitrogen potential of the surface to be coated is high and the rate of nitride is also high. Furthermore, because the surface to be treated is heated directly as a result of glow discharge, there is no need for external heating. Thus the treatment temperature is relatively low and there is less deformation. Because of these advantages, there is no need for pre-treatment to remove the passive layer from the surface when using the method for austenite-based stainless steel components that are required to be corrosion resistant and abrasion resistant. This makes the plasma-nitride technique an efficient method. Furthermore, although the surface layer becomes hard in the common method of nitride treatment of stainless steel at temperatures between 773 and $843 \mathrm{~K}$, nitrogen gets bonded to the chromium in the substrate, which reduces the concentration of chromium in the substrate, resulting in a reduction of corrosion resistance. In contrast, by conducting plasma nitride at lower temperatures between 673 and $723 \mathrm{~K}$, an S phase of improved quality is formed at the topmost portion of the surface, which provides excellent corrosion resistance [10-12]. In this study, the reason for our inability to confirm the formation of chromium nitride in the nitride phase may have been that the treatment was carried out at temperatures up to $723 \mathrm{~K}$.

\subsection{Hydrogen-Permeation Behavior}

The relationship between the crystalline grain size and hydrogen permeability at $773 \mathrm{~K}$ is shown in Fig. 5. Test specimens covered with films consisting of smaller crystalline grains tended to have lower hydrogen permeability. When the surface nitride 2 sample was set up on the hydrogen permeation testing apparatus, it was not possible to measure hydrogen permeation by easily by removing the hardened surface layer. Therefore, the hydrogen permeation test was carried out for the nitride surface 1 sample. The results are shown in Fig. 5. In all of the samples, the hydrogen permeation was found to be temperature dependent, i.e., the lower the temperature, the lower the hydrogen permeation. The hydrogen permeation of nitride-treated sample 1 was one order of magnitude less than that of the SUS316L substrate. The plasma nitride sample 1 was confirmed to have a hydrogen permeation prevention effect.

Under nitride treatment condition 1 , as mentioned above, a nitride phase mainly consisting of $\varepsilon-\mathrm{Fe}_{2-3} \mathrm{~N}$ and a nitrogen-dispersed phase was generated on the surface of the stainless steel, which was thought to effectively prevent hydrogen permeation.

From studies [13-17] related to the state of hydrogen in a substrate, lattice defects (atomic holes, transitions, grain boundaries), impurity atoms, deposition defects, inclusion interfaces, and voids are mentioned as hydrogen trapping sites. From the size of the trapping sites and the bonding energy between hydrogen atoms, it has recently become possible to

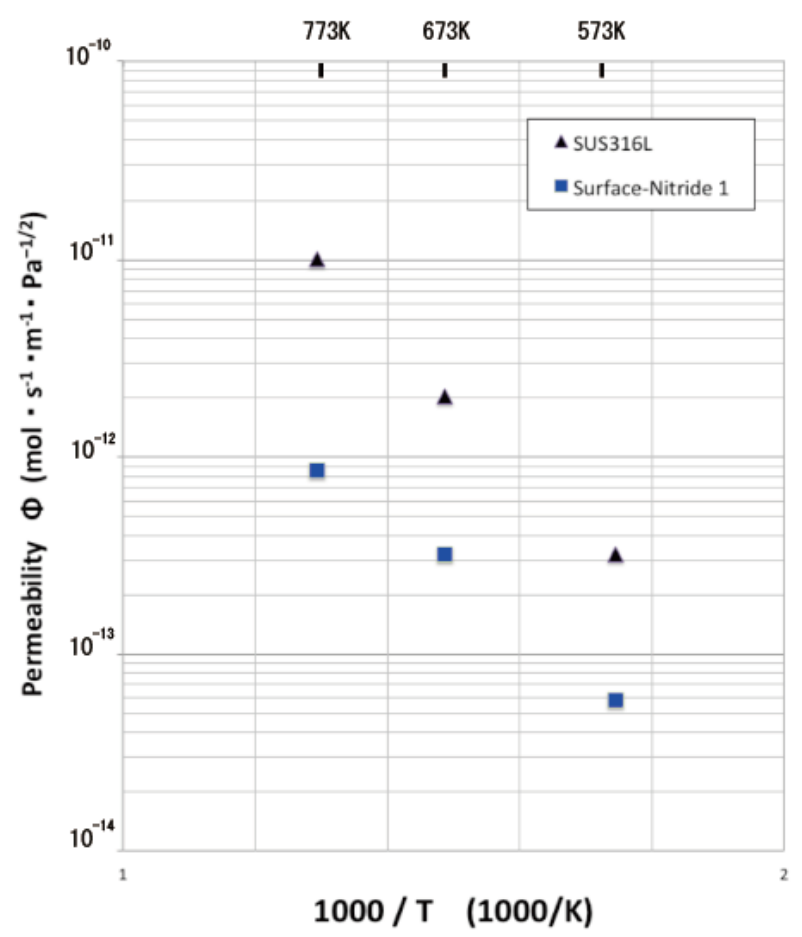

Fig. 5 Arrhenius plot of hydrogen permeability of samples as a function of temperature. 
separate the state of existence of hydrogen by means of thermal desorption spectrometry (TDS).

The embrittlement of iron and steel has been actively studied since the 1970s. Limiting the quantity of hydrogen in a crystal and lattice solid solubility is sought from the quantity of hydrogen in a sample of pure iron that is in equilibrium with a highly pure hydrogen gas atmosphere [13-15]. Solid solubility is the hydrogen atomic ratio corresponding to regular lattice points, but the concentration of hydrogen is known to be proportional to the square root of the hydrogen pressure. The solid solubility of hydrogen in iron and steel will be affected by the purity of the sample and the surface conditions, but the results from various researchers at $673 \mathrm{~K}$ and above are in fair agreement [13-15]. The temperature dependence is close to a linear Arrhenius plot. This is believed to be an essential phenomenon associated with the state of a solid solution such as the position occupied by hydrogen between lattices. Furthermore, the agreement of the data for various samples means that the quantity of hydrogen that can be absorbed by iron at $673 \mathrm{~K}$ and above is not affected much by crystal lattice defects such as impurities or crystal grain boundaries.

In the case of ion-covalent-bonded substances such as ceramics, the hydrogen dispersion in iron and steel is known to be several orders of magnitude less [1, 18, 19]. As regards iron nitride or a nitrogen-dispersed phase in the current study, the present paper does not give a full account of how or where the atomic hydrogen is dispersed and trapped; this is a topic for further research. However, hydrogen-trapping sites are supposed to be present in large numbers in the iron nitride and nitrogen-dispersed phase, forming a barrier to the dispersion of hydrogen and they are effective in manifesting such a barrier function.

\section{Conclusions}

The surface of austenitic stainless steel SUS316L was treated with nitrogen-plasma and its hydrogen permeability function was investigated. Plasma nitride was carried out using glow discharge in a vacuum chamber by connecting the stainless steel substrate to a biased power source. On the stainless steel surface, a nitride phase and a nitrogen-dispersed phase were found $5 \mu \mathrm{m}$ from the surface. The nitride phase was mainly consisting of $\varepsilon-\mathrm{Fe}_{2-3} \mathrm{~N}$ particles $0.1-0.4 \mu \mathrm{m}$ in size. It was confirmed that this nitride phase had hydrogen permeability an order of magnitude less than that for the untreated sample. The plasma nitride treatment can be applied for materials of complex shapes, the inner surfaces of pipes, or medium/large-sized components used in hydrogen atmosphere.

\section{References}

[1] Hollenberg, G. W., Simonen, E. P., Kalinin, G., and Terlain, A. 1995. "Tritium/Hydrogen Barrier Development.” Fusion Eng. Des. 28: 190-208.

[2] Checcketto, R., Bonelli, M., Gratton, L. M., Miotello, A., Sabbioni, A., and Guzman, L. et al. 1996. "Analysis of the Hydrogen Permeation Properties of TiN-TC Bilayers Deposited on Martensitic Stainless Steel." Surf. Coat. Technol. 83: 40-4.

[3] Tamura, M., Noma, M., and Yamashita, M. 2014. "Characteristic Change of Hydrogen Permeation in Stainless Steel Plate by BN Coating." Surf. Coat. Technol. 260: 148-54.

[4] Tamura, M., and Shibata, K. 2005. "Evaluation of Mechanical Properties of Metals at $45 \mathrm{MPa}$ Hydrogen." J. Jpn. Inst. Met. 69: 1039-48.

[5] Masaki, K., and Ochi, Y. 2008. "Effect of Low Temperature Gas Nitriding and Low Temperature Gas Carburizing on High Cycle Fatigue Property in SUS316L.” J. Soc. Mater. Sci., Jpn. 57 (6): 563-8.

[6] Stern, S. A. 1968. “The 'Barrer' Permeability Unit." $J$. Polym. Sci. Part A-2: Polymer Physics 6 (11): 1933-4.

[7] JIS K7126-2: 2006 (Japanese Industrial Standards Committee).

[8] ISO15105-1: 2007 (International Organization for Standardization).

[9] Chikada, T., Suzuki, A., Yao, Z., Levchuk, D., Mainer, H. and Terai, T. et al. 2009. "Deuterium Permeation Behavior of Erbium Oxide Coating on Austenitic, Ferritic, and Ferritic/Martensitic Steels." Fusion Eng. Des. 84 (2-6): 590-2.

[10] Thaiwatthana, S., Li, X. Y., Dong, H., and Bell, T. 2002. "Comparison Studies on Properties of Nitrogen and Carbon S Phase on Low Temperature Plasma Alloyed 
AISI 316 Stainless Steel.” Surf. Eng. 18 (6) 433-7.

[11] Sun, Y., and Bell, T. 2002. "Residual Stress Evolution and Relaxation in Carbon S Phase Layers on AISI 316 Austenitic Stainless Steel.” Surf. Eng. 18 (6): 443-7.

[12] Gemma, K., and Kawakami, K. 1988. "Abnormal Temperature Dependence of Nitriding Rate in the Austenitic Stainless Steels.” J. Japan Inst. Metals 52 (7): 701-10.

[13] Beachem, C. D. 1972. "A New Model for Hydrogen-Assisted Cracking (Hydrogen Embrittlement)." Metall. Trans. 3: 437-51.

[14] Nagumo, M., Nakamura, M., and Takai, K. 2001. "Hydrogen Thermal Desorption Relevant to Delayed-Fracture Susceptibility of High-Strength Steels." Metall. Trans. A 32A: 339-47.
[15] Takai, K., Homma, Y., Izutsu, K., and Nagumo, M. 1996. J. Jpn. Inst. Met. 30: 1155-62.

[16] A. Oudriss, J. Creus, J. Bouhattate, E. Conforto, C. Berziou, C. Savall, and Feaugas, X. 2012. "Grain Size and Grain-Boundary Effects on Diffusion and Trapping of Hydrogen in Pure Nickel." Acta Materialia 60 (19) 6814-28.

[17] Yazdipour, N., Dunne, D., and Perelome, E. 2012. "Effect of Grain Size on the Hydrogen Diffusion Process in Steel Using Cellular Automaton Approach." Mater. Sci. Forum 706-709: 1568-73.

[18] Yamabe, J., Matsuoka, S., and Murakami, Y. 2013. "Surface Coating with a High Resistance to Hydrogen Entry under High-Pressure Hydrogen-Gas Environment." Int. J. Hydrogen Energy 38: 10141-54. 